\title{
Anwendung eines thermoelektrischen Gassensors zur Erfassung reduzierender Komponenten in Verbrennungsabgasen von Kleinfeuerungsanlagen
}

\author{
Gunter Hagen ${ }^{1}$, Binayak Ojha ${ }^{2}$, Julia Wohlrab' ${ }^{1}$, Heinz Kohler ${ }^{2}$, Ralf Moos ${ }^{1}$ \\ ${ }^{1}$ Lehrstuhl für Funktionsmaterialien, Universität Bayreuth, 95440 Bayreuth, Deutschland \\ ${ }^{2}$ Institut für Sensor- und Informationssysteme, Hochschule Karlsruhe, 76133 Karlsruhe, Deutschland \\ gunter.hagen@uni-bayreuth.de
}

\begin{abstract}
Zusammenfassung
Durch Regelung der Verbrennungsluft in mit Scheitholz befeuerten Anlagen kleiner Leistung kann deren Schadstoffausstoß erheblich gesenkt werden, insbesondere, wenn neben der Verbrennungstemperatur und der Sauerstoffkonzentration im Abgas auch der Anteil reduzierender Gase $(\mathrm{CO} / \mathrm{HC})$ im Verbrennungsluft-Regelungsverfahren berücksichtigt wird. Gassensoren müssen für diese Anwendung besonders hochtemperaturstabil und alterungsresistent sein. Thermoelektrische Sensoren erfüllen diese Anforderungen. Sie erfassen eine Temperaturdifferenz zwischen einem inerten und einem katalytisch beschichteten Bereich auf der Sensoroberfläche und damit die entstehende Exothermie am Katalysator bei Beprobung mit reduzierenden Gasen. Für die notwendige Aktivität des Katalysators sorgt ein auf der Sensorrückseite aufgebrachter Dickschicht-Heizer, mit dem die absolute Sensortemperatur eingestellt und konstant gehalten wird. Solche Sensoren können in keramischer Dickschichttechnik mit abgasstabilen Materialien hergestellt werden. Messungen im Rauchgas während realer Abbrände zeigen das Potenzial der Anwendung thermoelektrischer Sensoren. Die Signale werden durch simultane Gasanalytik mittels Hochtemperatur-FTIR referenziert. Dafür wurden die Hauptkomponenten, die bei der Verbrennung entstehen, gemäß ihres zu erwartenden Beitrags zur Exothermie am Sensor aufsummiert. Das Sensorsignal, das nur um einen Offset-Wert korrigiert wurde, zeigt eine sehr gute Übereinstimmung mit diesen Daten.
\end{abstract}

Keywords: Holzverbrennung, Thermoelektrischer Gassensor, FTIR, CO-Äquivalent $\left(\mathrm{CO}_{e}\right)$

\begin{abstract}
Motivation
Kaminöfen und Kleinfeuerungsanlagen erfreuen sich großer Beliebtheit. Allerdings tragen diese nicht unwesentlich zur Verunreinigung der Luft bei. Insbesondere der Ausstoß von mit Organik beladenen Feinstaubpartikeln und die Emissionen von Kohlenmonoxid (CO) oder von unverbrannten Kohlenwasserstoffen (HC) stellen ein Problem dar.

Jüngste Arbeiten zeigen, dass durch Regelung der Verbrennungsluft der Schadstoffausstoß solcher Anlagen erheblich gesenkt werden kann. Für ein besonders effizientes Verbrennungsluft-Regelungsverfahren ist dafür aber neben der Verbrennungstemperatur und der Restsauerstoffkonzentration im Abgas auch der Anteil reduzierender Gase (CO/HC) während des Betriebs zu berücksichtigen [1,2]. Die Verfügbarkeit geeigneter Gassensoren spielt deshalb eine wesentliche Rolle [3].

Gassensoren müssen für diese Anwendung besonderen Anforderungen genügen. Das
\end{abstract}

Rauchgas enthält nicht nur Komponenten, die im Allgemeinen als "Sensorgifte" bezeichnet werden (so z.B. $\mathrm{SO}_{2}$ o.ä.). Auch Partikel (Rußoder Asche) können den Sensor schädigen. Deshalb werden Sensoren bei hohen Temperaturen betrieben, um Ablagerungen auf der Sensoroberfläche zu vermeiden. Allerdings heißt dies auch, dass Gassensoren dann besonders hochtemperaturstabil und bei der Einsatztemperatur auch alterungsresistent sein müssen.

Viele Sensorprinzipien scheitern genau an dieser Vorgabe, da beispielsweise eine schleichende Veränderung der Morphologie (Versinterung) zu einer Änderung des Grundwiderstands führt (Drift bei resistiven Sensoren) oder dass das Elektrodenmaterial der Temperaturbelastung oder Atmosphäre nicht langfristig standhält (Alterung oder Vergiftung bei elektrochemischen Mischpotenzial-Sensoren) [4-6].

Besser geeignet scheint die Anwendung des thermoelektrischen Prinzips. Das Messsignal 
solcher Sensoren ist eine Thermospannung. Sie entsteht durch den Temperaturunterschied zwischen zwei Bereichen des Sensorbauteils.

\section{Thermoelektrische Gassensoren}

Thermoelektrische Sensoren erfassen eine Temperaturdifferenz zwischen einem inerten und einem katalytisch beschichteten Bereich auf der Sensoroberfläche und demnach die entstehende Exothermie am Katalysator bei Beprobung mit reduzierenden Gasen [7]. Für die Aktivität des Katalysators sorgt ein auf der Sensorrückseite aufgebrachter DickschichtHeizer, mit dem die absolute Sensortemperatur (z.B. $600{ }^{\circ} \mathrm{C}$ ) eingestellt und konstant gehalten wird. Die Herstellung solcher Sensorbauteile ist in einfacher Dickschichttechnik unter Verwendung abgasstabiler Materialien möglich. Das gut zu verstehende Sensorprinzip ermöglicht eine vollständige Beschreibung des Sensorverhaltens durch ein FEM-Modell [8]. Damit können auch weitere Entwicklungen zur Erhöhung der Empfindlichkeit oder Robustheit unterstützt werden (siehe z.B. [9]).

\section{Experimentelles}

Ein thermoelektrischer Sensor auf Basis eines Aluminiumoxid-Substrates mit einer Thermopile-Struktur aus $\mathrm{Pt}$ und Pt-Rh Dickschicht-Leiterbahnen [10] wurde zunächst im synthetischen Abgas im Labor charakterisiert. Als Katalysator wurde eine Dickschicht aus 1 gew\% $\mathrm{Pt}$ auf $\mathrm{Al}_{2} \mathrm{O}_{3}$ verwendet. Der Sensor hatte eine Betriebstemperatur von $600{ }^{\circ} \mathrm{C}$. Für $\mathrm{CO}$, Methan $\left(\mathrm{CH}_{4}\right)$, Propen $\left(\mathrm{C}_{3} \mathrm{H}_{6}\right)$ und Propan $\left(\mathrm{C}_{3} \mathrm{H}_{8}\right)$ ergeben sich lineare Kennlinien, d.h. eine Erhöhung der Konzentration führt direkt zu vermehrter Reaktionswärme am Katalysator und dementsprechend $z u$ einer höheren Temperaturdifferenz bzw. steigendem Sensorsignal. Die Kennlinien-Steigung (Empfindlichkeit $S$ ) hängt dabei nicht nur vom Heizwert der Gase, sondern auch von den Diffusions- und Reaktionsbedingungen an der Katalysatorschicht ab. Bezieht man die Empfindlichkeiten $S$ auf das dem CO äquivalente Signal, dann ergeben sich folgende Zusammenhänge: $\mathrm{S}_{\mathrm{CH} 4}=0,5 \times \mathrm{S}_{\mathrm{CO}}$, $\mathrm{S}_{\mathrm{C} 3 \mathrm{H} 6}=15 \times \mathrm{S}_{\mathrm{CO}}, \mathrm{S}_{\mathrm{C} 3 \mathrm{H} 8}=4 \times \mathrm{S}_{\mathrm{CO}}$.
Im zweiten Schritt erfolgte die Charakterisierung des Sensors im Realabgas einer mit Stückholz befeuerten Einzelraumfeuerstätte (SF10SK, Brunner $\mathrm{GmbH}$ ). Hier wurde das Abgas während eines kompletten Abbrandes mittels HT-FTIR in Intervallen von 10s analysiert. Die Hauptkomponenten der Emission sind $\mathrm{CO}$, Methan $\left(\mathrm{CH}_{4}\right)$, Ethen $\left(\mathrm{C}_{2} \mathrm{H}_{4}\right)$ und weitere Kohlenwasserstoffe (gemessen als Summen-HC (THC)). Deren gemessene Konzentrationen wurden zu einem CO-äquivalenten Summensignal $c\left(\mathrm{CO}_{\mathrm{e}}\right)$ umgerechnet. Dabei kommen die o.g. Gewichtungsfaktoren zum Tragen, die den Beitrag der jeweiligen Gase zur Exothermie am Sensor abbilden. Dabei wird für Ethen ein dem Propen ähnlicher Wert von 13, für THC der Wert 5 gewählt. Die resultierende Kurve wird nun mit dem gemessenen Sensorsignal verglichen. Der Sensor wurde auch hier bei $600{ }^{\circ} \mathrm{C}$ in einer Einbauposition im Kamin betrieben.

\section{Vergleich des Sensorsignals mit FTIR- Daten}

Das Sensorsignal zeigt eine sehr deutliche Übereinstimmung mit dem gewichteten Summensignal der reduzierenden AbgasAnteile, die mittels FTIR gemessen wurden (Abbildung 1). Dabei ist besonders darauf hinzuweisen, dass die aufgetragene Sensorspannung das gemessene Rohsignal wiedergibt, das lediglich um einen Offset-Wert zu Beginn der Messung $(t=0)$ korrigiert wurde. Es zeigt sich ein schnelles Ansprechen auf Änderungen in der Gasatmosphäre. Alle Spitzen, die in den summierten FTIR-Daten auftreten, werden auch vom Sensor erkannt. Je 1000 ppm COe liefert der Sensor ein Signal von $10 \mu \mathrm{V}$.

\section{Ausblick}

Thermoelektrische Sensoren besitzen großes Potenzial für die Anwendung bei der Verbrennungsluftregelung in der BiomasseVerbrennung. Zukünftige Herausforderung ist die Zuordnung der Signale zu bestimmten Gasspezies, was durch Variation der Sensortemperatur erreicht werden kann [11]. 


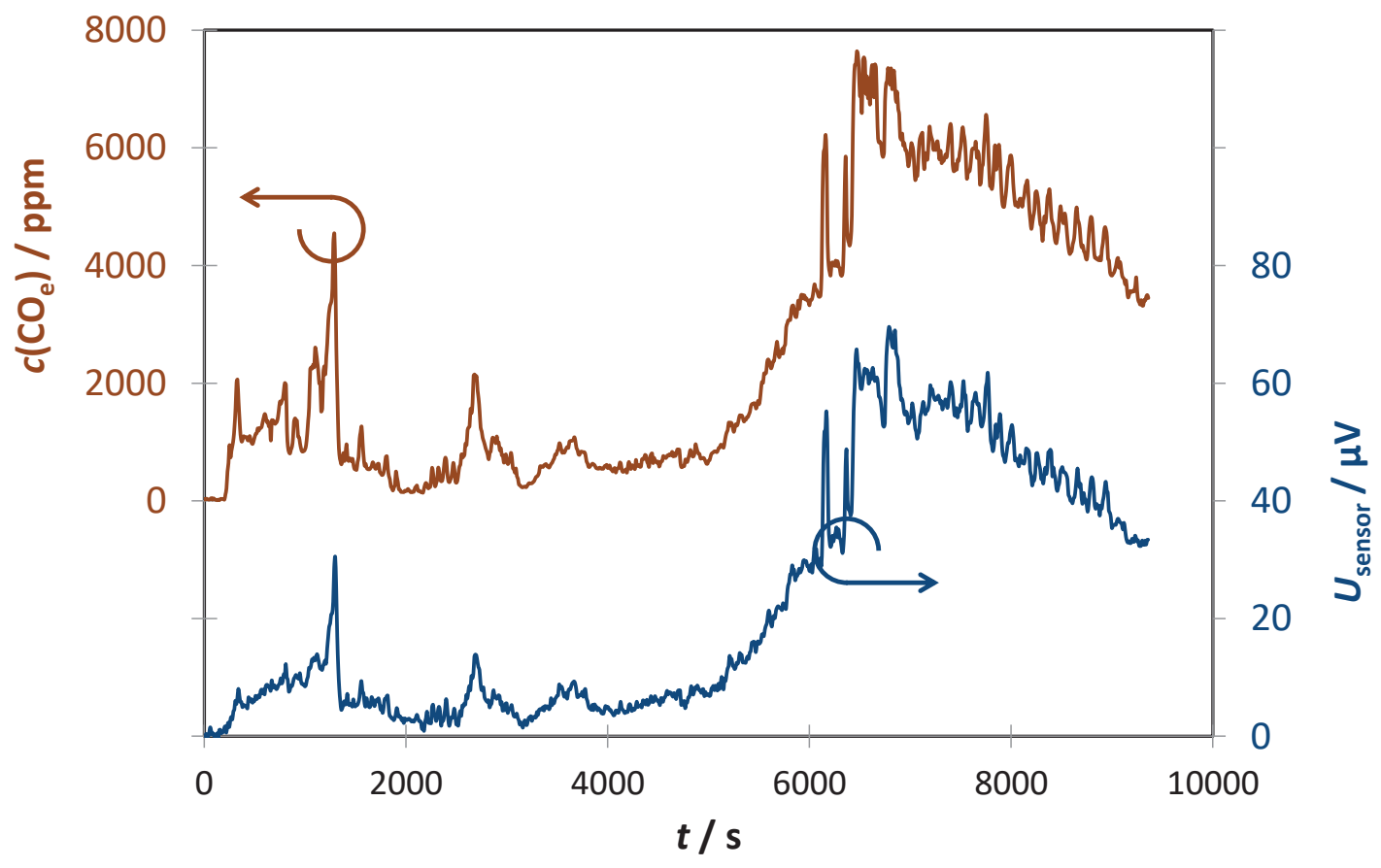

Abb. 1: Vergleich zwischen Sensor-Rohsignal (korrigiert um den Wert bei $t=0$ ) und Summe der Konzentrationen der Hauptkomponenten - gewichtet nach ihrem zu erwartenden Beitrag zum Sensorsignal - c(COe), gemessen mit FTIR während eines Abbrandes.

\section{Literaturnachweis}

[1] P. Butschbach, F. Hammer, H. Kohler, A. Potreck, Th. Trautmann, Sensors and Actuators B Chemical 137 (1), 32-41 (2009); doi: 10.1016/j.snb.2008.12.007

[2] B. Ojha, N. Illyaskutty, J. Knoblauch, M.R. Balachandran, H. Kohler, Journal of Sensors and Sensor Systems 6, 237-246 (2017); doi: 10.5194/jsss-6-237-2017

[3] H. Kohler, B. Ojha, N. Illyaskutty, I. Hartmann, C. Thiel, K. Eisinger, M. Dambacher; Journal of Sensors and Sensor Systems, submitted

[4] Moos, R.: Recent Developments in Automotive Exhaust Gas Sensing, Proceedings SENSOR 2009 I, 227-231 (2009), doi: 10.5162/sensor09/v1/b5.1

[5] J. Riegel, H. Neumann, H.-M. Wiedenmann, Solid State Ionics 152-153, 783-800 (2002), doi: 10.1016/S0167-2738(02)00329-6
[6] R. Moos, K. Sahner, M. Fleischer, U. Guth, N. Barsan, U. Weimar, Sensors 9 (6), 4323-4365 (2009); doi: 10.3390/s90604323

[7] S. Wiegärtner, G. Hagen, J. Kita, W. Reitmeier, M. Hien, P. Grass, R. Moos, Sensors and Actuators B Chemical 21, 234240 (2015); doi: 10.1016/j.snb.2015.02.083

[8] T. Ritter, S. Wiegärtner, G. Hagen, R. Moos, Proceedings SENSOR 2017, P5.7 (2017); doi: 10.5162/sensor2017/P5.7 or Journal of Sensors and Sensor Systems, submitted

[9] J. Kita, G. Hagen, C. Schmidt, R. Moos, Proceedings 1(4), 403 (2017); doi: 10.3390/proceedings 1040403

[10] J. Kita, S. Wiegärtner, R. Moos, P. Weigand, A. Pliscott, M.H. LaBranche, H.D. Glicksman, Procedia Engineering 120, 828-831 (2015); doi: 10.1016/j.proeng.2015.08.692

[11] G. Hagen, N. Leupold, S. Wiegärtner, R. Moos, Topics in Catalysis 60, 312-317 (2017); doi: 10.1007/s11244-016-0617-8 